\title{
Localization of Autophagosome in Porcine Follicular Cumulus-oocyte Complex
}

\author{
Seunghoon Lee, Dong-Hoon Kim, Gi-Sun Im, Sun-A Ock, Imran Ullah and Tai-Young Hur ${ }^{\dagger}$
}

National Institute of Animal Science, RDA, Wanju 55365, Republic of Korea

\begin{abstract}
Autophagy is an intracellular degradation and recycling system. Oocyte maturation is dynamic process, in which various proteins should be synthesized and degraded. In our previous study, we reported the loci of autophagosome and dynamics of autophagic activity in porcine oocytes during in vitro maturation. In this study, we verified loci of autophagosome in porcine follicular cumulus-oocyte complex by detection of microtubule-associated protein 1A/1B-light chain 3 (LC3) which is the reliable marker of autophagosome. Porcine ovary including various sizes of follicles was fixed within 1 hour after collection from slaughterhouse. After fixation, immunohistochemistry was conducted on sliced ovary tissue containing various sizes of follicles by using LC3 antibody. As a result, LC3 signal was clearly detected in both cumulus and oocytes of various sizes of follicles. We also found ring shaped signal which represent autophagosome near oocyte membrane. Most of the signals in oocytes were localized nearby cellular membrane while evenly dispersed in cumulus cells. Therefore, this result suggests that autophagy occurs in porcine COCs (cumulus-oocyte complexes) at follicular stage.
\end{abstract}

(Keywords: Autophagosome, Porcine, Follicle, LC3, Localizaton)

\section{Introduction}

Microtubule-associated protein 1A/1B light chain 3(LC3)-II is the most reliable marker of autophagosome because they exist on the autophagosome membrane during the entire autophagosome life cycle (Mizushima 2004). LC3-I is cytosolic type of LC3 while LC3-II is its membrane-embedded form (Fritzen et al. 2016). Therefore, LC3-II is used as a potent autophagosome marker which represents autophagic activity. Autophagosome is a kind of unit of entire autophagy system. In autophagy system, unfolded useless protein and old cellular components are packed by autophagosome. Subsequently, lysosome fuse with autophagosome and inside contents of autophagosome are degraded by hydrolytic enzyme of lysosome (Yin et al. 2016). Although there is another type of autophagy i.e. micro autophagy or chaperone-mediated autophagy, normally macro autophagy is termed as autophagy which related to fusion between autophagosome and lysosome (Feng et al. 2014; Sahu et al. 2011; Tang et al. 2017). Autophagy is generally activated when cellular stress is increased by cellular alteration like embryo development or insect metamorphosis (Mizushima and Komatsu 2011;
Nakashima et al. 2017). Autophagy helps cellular alteration as they are engaged to degrade and recycle useless protein or cellular components (Masclaux-Daubresse et al. 2017). Oocyte maturation is also cellular alteration process, likewise cytosol of oocyte should be grown dramatically or meiosis should be occurred just in short period which is follicular stage (Miyano and Manabe 2007). We focused on this kind of autophagy character and hypothesized that autophagy is activated during oocyte maturation. In our previous study, we elucidated the dynamics of autophagic activity during in vitro maturation of porcine oocytes (Lee et al. 2014). However, we suspected that only in vitro circumstance activate autophagy. Therefore, in this study to confirm autophagy in porcine oocytes at follicular stage, we evaluated autophagosome localization by immunohistochemistry of porcine ovary using LC3 antibody.

\section{MATERIALS and METHODS}

\section{Immunohistochemistry of porcine ovary tissue}

Porcine ovaries in $0.9 \%$ saline solution were moved to laboratory from slaughterhouse within 1 hour. After 3 times

\footnotetext{
† Correspondence: Tai-Young Hur, Ph.D.

Phone: +82-63-238-7271

E-mail: tyohur@korea.kr
} 
washing in phosphate buffered saline(PBS), ovary was cut by $5 \times 5 \mathrm{~mm}$ size and every pieces of ovary tissue fixed in $10 \%$ formaldehyde neutral buffer solution (Nacalai Tesque, Kyoto, Japan). After 48 hours fixation at room temperature, each piece of ovary was embedded in paraffin wax and sectioned with $5 \mu \mathrm{m}$ thickness. Sectioned samples of ovary tissue were placed on slide glass and dried at room temperature. Then, samples were deparaffinized and incubated with HistoVT One (Nacalai Tesque) at $90^{\circ} \mathrm{C}$ for 30 minute. After washing, samples were proceeded to blocking step by treatment with Blocking One (Nacalai Tesque) at $4^{\circ} \mathrm{C}$ for 1 hour. Subsequently, samples were incubated with rabbit anti-LC3 monoclonal antibody (dilution 1:200; Cell signaling, Danvers, MA) overnight at $4{ }^{\circ} \mathrm{C}$. Furthermore, samples were incubated with Alexa Fluor 488-labeled anti-rabbit secondary antibody

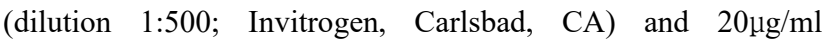
propidium iodide (PI) (Sigma Chemical, St Louis, MO) at $4{ }^{\circ} \mathrm{C}$ for 2 hours. After washing in PBS, samples were observed under a LSM700 confocal scanning laser microscope (Zeiss, Feldbach, Switzerland).

\section{RESULTS and DISCUSSION}

Autophagy is well conserved cellular degradation system. Autophagy is generally known to have a role of stabilizing homeostasis and protection against various stresses. Although most autophagy researches are focused on cancer researches, reproductive researches have also investigated autophagy. Main focus of autophagy in reproductive studies is related to relationship between apoptosis and autophagy against various stresses like oxidative stress, toxic stress, and so on (Han et al. 2016; Wang et al. 2016; Zhang et al. 2016). In animal reproductive studies, autophagy was well known to be activated in embryo stages (Jin et al. 2016; Xu et al. 2012). However, only few studies about autophagy in animal unfertilized oocytes have conducted. In our previous study, we reported that autophagic activity is dynamic during in vitro maturation of porcine COCs (Lee et al. 2014). In this study, we examine location of autophagosome in porcine follicular COCs to verify autophagy work in porcine follicular oocytes. Immunohistochemistry analysis in various sizes of follicles whose diameter are $\leq 1 \mathrm{~mm}, 1 \sim 2 \mathrm{~mm}$, and $\geq 2 \mathrm{~mm}$ showed LC3 expression in both cumulus cell and oocyte, regardless of their follicular sizes (Fig1. A, B, C). We classified follicular size with 3 groups. $\geq 2 \mathrm{~mm}$ of follicle represent almost completed maturation, while $1 \sim 2 \mathrm{~mm}$ of follicle represent maturating phase and $\leq 1 \mathrm{~mm}$ of follicle represent immature phase, respectively. Especially, clear dot- and ring- shaped LC3 signal was shown near plasma membrane in magnified picture of $2 \mathrm{~mm}$ follicular COCs (Fig1. D). We employed LC3 antibody as a marker of autophagosome, because among various autophagy marker like beclin1 or autophagy related genes(ATG) family, only LC3-II exist autophagosome membrane in whole autophagy steps which are made of nucleation, elongation, completion, and degradation (Tam et al. 2017). For example, beclin1 works only early stage of autophagy of nucleation step then it release to cytosol (Fimia et al. 2011). Therefore, beclin1 is suitable for only early stage of autophagy while LC3-II is able to represent autophagy activity. However, LC3 antibody detect both LC3-I(cytosolic form) and LC3-II(membrane form) in which phosphatidyletanolamine(PE) bound to LC3-I (McLeland et al. 2011). In western blotting, it is easy to distinguish between LC3-I and LC3-II because of their different molecular size. In immunostaining, LC3-II is known to form dot- shape at nucleation step of autophagy, cup- shape at elongation step, and ring- shape at completion and degradation steps, but not LC3-I (Pinar et al. 2013; Yano et al. 2008). In our magnified picture, we detected dot- and ring- shaped LC3 signal. This means that these dot- and ring- shaped LC3 signal represent LC3-II which is on autophagosome membrane. Conclusively, our data verified that autophagosome exit in porcine follicular COCs. In other words, autophagy works in porcine follicular COCs. Autophagy is normally activated when a cell was forced to dramatic internal change (Nakashima et al. 2017). In this circumstance, when cell stress is increased by stimulation, autophagy has two of paradoxical roles. First role is to detect a cell as they participate in keep on homeostasis by degrading and recycling of useless unfolded proteins and cellular component (Zou et al. 2017), while another role is to induce cell death with apoptosis (Qian et al. 2017). Until ovulation, porcine oocyte in follicular stage has to change dramatically including dramatic cytosolic growth. It is possible that autophagy participates in cytosolic growth by degrading and recycling gap junction or imported materials via gap junction. In other cell, it is reported that connexin 43 and connexin 37, which are main component of gap junction was degraded by autophagy (Carette et al. 2015; Sun et al. 2015). Our data also showed that autophagosomes 

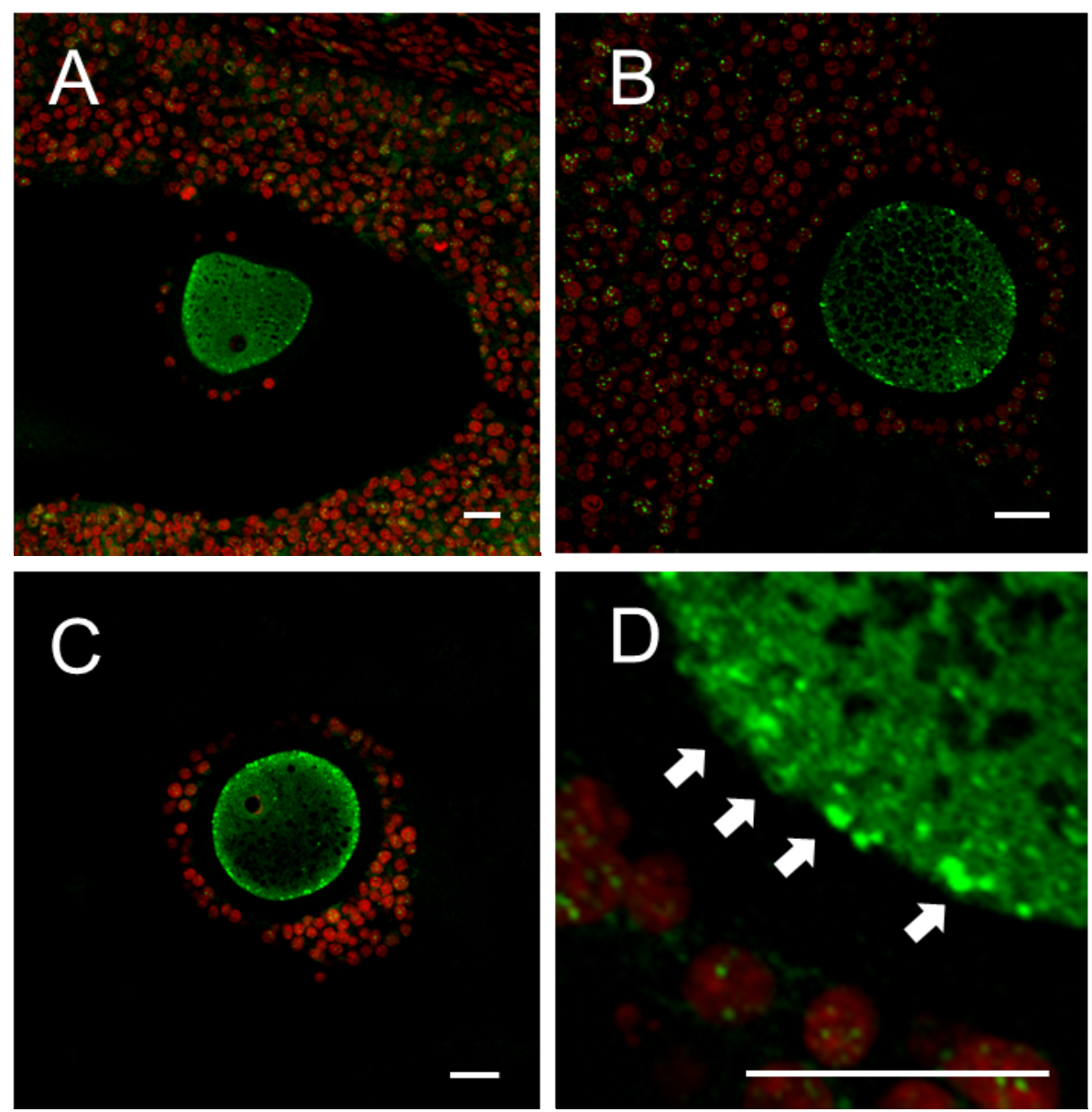

Figure 1. Immunohistochemistry analysis of microtubule-associated protein light chain 3 in the porcine ovary. Tissues were collected from porcine ovaries. Fixation was performed in $10 \%$ formaldehyde neutral buffer solution. The samples were then embedded in paraffin and cut to $5 \mathrm{~mm}$. The cumulus cells and oocytes in follicles of sizes of $\leq 1 \mathrm{~mm}(A), 1 \sim 2 \mathrm{~mm}(B)$, and $\geq 2 \mathrm{~mm}(C)$ were photographed under a confocal scanning laser microscope. The picture of over $2 \mathrm{~mm}$ follicle (C) was obtained under magnification. Arrows indicate dot- or ring- shaped LC3 immunoreactive signals (D). The green and red indicate microtubule-associated protein light chain 3 (LC3) and the nucleus, respectively. Scale bar $=20 \mu \mathrm{m}$.

mostly exist near plasma membrane in oocytes. Recently, reports about autophagy role in reproduction have been increased. In mouse, autophagic activity is highly regulated from embryogenesis but not in oogenesis or follicular genesis (Nakashima et al. 2017). LC3 signal was detected in only rat cumulus cell but not rat oocyte in immunohistochemistry (Choi et al. 2010). However, during in vitro maturation of porcine immature oocyte, autophagic dynamics has been confirmed (Lee et al. 2014). For better understanding of porcine oocyte maturation mechanism related to autophagy, further studies are needed to examine autophagy role in follicular oocyte maturation. In this study, we showed that location of autophagosome in porcine follicular COCs for the first time and suggested the possibility that autophagy works in follicular COCs. This study will provide fundamental data for future studies about the relationship between autophagy and oocyte maturation.

\section{ACKNOWLEDGMENTS}

This work was supported by a fund of Project No.PJ01092802 from Rural Development Administration, Republic of Korea. 


\section{REFERENCES}

Crette D, Gilleron J, Denizot JP, Grant K, Pointis G, and Segretain D. 2015. New cellular mechanisms of gap junction degradation and recycling. Biology of the cell 107:218-231.

Choi JY, Jo MW, Lee EY, Yoon BK, and Choi DS. 2010. The role of autophagy in follicular development and atresia in rat granulosa cells. Fertility and sterility 93:2532-2537.

Feng Y, He D, Yao Z, and Klionsky DJ. 2014. The machinery of macroautophagy. Cell research 24:24-41.

Fimia GM, Di Bartolomeo S, Piacentini M, and Cecconi F. 2011. Unleashing the Ambra1-Beclin 1 complex from dynein chains: Ulk1 sets Ambral free to induce autophagy. Autophagy 7:115-117.

Fritzen AM, Frosig C, Jeppesen J, Jensen TE, Lundsgaard AM, Serup AK, Schjerling P, Proud CG, Richter EA, and Kiens B. 2016. Role of AMPK in regulation of LC3 lipidation as a marker of autophagy in skeletal muscle. Cellular signalling 28:663-674.

Han J, Wang QC, Zhu CC, Liu J, Zhang Y, Cui XS, Kim NH, and Sun SC. 2016. Deoxynivalenol exposure induces autophagy/apoptosis and epigenetic modification changes during porcine oocyte maturation. Toxicology and applied pharmacology 300:70-76.

Jin YX, Zheng Z, Yu XF, Zhang JB, Namgoong S, Cui XS, Hyun SH, and Kim NH. 2016. Autophagy and ubiquitin-mediated proteolysis may not be involved in the degradation of spermatozoon mitochondria in mouse and porcine early embryos. Zygote (Cambridge, England) 24:31-41

Lee S, Hiradate Y, Hoshino Y, Tanemura K, and Sato E. 2014. Quantitative analysis in LC3-II protein in vitro maturation of porcine oocyte. Zygote (Cambridge, England) 22:404-410.

Masclaux-Daubresse C, Chen Q, and Have M. 2017. Regulation of nutrient recycling via autophagy. Current opinion in plant biology 39:8-17.

McLeland CB, Rodriguez J, and Stern ST. 2011. Autophagy monitoring assay: qualitative analysis of MAP LC3-I to II conversion by immunoblot. Methods in molecular biology (Clifton, NJ) 697:199-206.

Miyano T, and Manabe N. 2007. Oocyte growth and acquisition of meiotic competence. Society of Reproduction and Fertility supplement 63:531-538.

Mizushima N. 2004. Methods for monitoring autophagy. The international journal of biochemistry \& cell biology $36: 2491-2502$

Mizushima N, and Komatsu M. 2011. Autophagy: renovation of cells and tissues. Cell 147:728-741.

Nakashima A, Aoki A, Kusabiraki T, Shima T, Yoshino O, Cheng SB, Sharma S, and Saito S. 2017. Role of autophagy in oocytogenesis, embryogenesis, implantation, and pathophysiology of pre-eclampsia. The journal of obstetrics and gynaecology research 43:633-643.

Pinar M, Pantazopoulou A, and Penalva MA. 2013. Live-cell imaging of Aspergillus nidulans autophagy: RAB1 dependence, Golgi independence and ER involvement. Autophagy 9:1024-1043.

Qian HR, Shi ZQ, Zhu HP, Gu LH, Wang XF, and Yang Y. 2017. Interplay between apoptosis and autophagy in colorectal cancer. Oncotarget.

Sahu R, Kaushik S, Clement CC, Cannizzo ES, Scharf B, Follenzi A, Potolicchio I, Nieves E, Cuervo AM, and Santambrogio L. 2011. Microautophagy of cytosolic proteins by late endosomes. Developmental cell 20:131-139.

Sun LQ, Gao JL, Cui Y, Zhao MM, Jing XB, Li R, Tian YX, Cui JZ, and Wu ZX. 2015. Neuronic autophagy contributes to p-connexin 43 degradation in hippocampal astrocytes following traumatic brain injury in rats. Molecular medicine reports 11:4419-4423.

Tam SY, Wu VW, and Law HK. 2017. Influence of autophagy on the efficacy of radiotherapy. Radiation oncology (London, England) 12:57.

Tang Y, Wang XW, Liu ZH, Sun YM, Tang YX, and Zhou DH. 2017. Chaperone-mediated autophagy substrate proteins in cancer. Oncotarget.

Wang T, Han J, Duan X, Xiong B, Cui XS, Kim NH, Liu HL, and Sun SC. 2016. The toxic effects and possible mechanisms of Bisphenol A on oocyte maturation of porcine in vitro. Oncotarget 7:32554-32565.

Xu YN, Shen XH, Lee SE, Kwon JS, Kim DJ, Heo YT, Cui XS, and Kim NH. 2012. Autophagy influences maternal mRNA degradation and apoptosis in porcine parthenotes developing in vitro. The Journal of reproduction and development 58:576-584.

Yano T, Mita S, Ohmori H, Oshima Y, Fujimoto Y, Ueda R, Takada H, Goldman WE, Fukase K, Silverman N et al., 2008. 
Autophagic control of listeria through intracellular innate immune recognition in drosophila. Nature immunology 9:908-916.

Yin Z, Pascual C, and Klionsky DJ. 2016. Autophagy: machinery and regulation. Microbial cell (Graz, Austria) 3:588-596.

Zhang Y, Han J, Zhu CC, Tang F, Cui XS, Kim NH, and Sun SC. 2016. Exposure to HT-2 toxin causes oxidative stress induced apoptosis/autophagy in porcine oocytes. Scientific reports 6:33904.
Zou Q, Tan S, Yang Z, Zhan Q, Jin H, Xian J, Zhang S, Yang L, Wang L, and Zhang L. 2017. NPM1 Mutant Mediated PML Delocalization and Stabilization Enhances Autophagy and Cell Survival in Leukemic Cells. Theranostics 7:2289-2304.

Received August 16 2017, Revised September 13 2017, Accepted September 18, 2017 
